\title{
HOMOTOPY PERTURBATION TRANSFORM METHOD FOR SOLVING THE PARTIAL AND THE TIME-FRACTIONAL DIFFERENTIAL EQUATIONS WITH VARIABLE COEFFICIENTS
}

\section{ABDELKADER KEHAILI, ALI HAKEM AND ABDELKADER BENALI}

(Received 8 July 2019; Revision Accepted 20 September 2019)

\begin{abstract}
In this paper, we present the exact solutions of the Parabolic-like equations and Hyperbolic-like equations with variable coefficients, by using Homotopy perturbation transform method (HPTM). Finally, we extend the results to the time-fractional differential equations.
\end{abstract}

KEYWORDS: Caputo's fractional derivative, fractional differential equations, homotopy perturbation transform method, hyperbolic-like equation, Laplace transform, parabolic-like equation.

MSC 2010: 35L15, 74G10, 34A08, 35K15.

\section{INTRODUCTION}

The Parabolic-like and Hyperbolic-like equations can be used to describe wide variety of phenomena such as sound, heat, diffusion, electrostatics, electrodynamics, fluid dynamics, elasticity, or quantum mechanics. These seemingly distinct physical phenomena can be formalized similarly in terms of Parabolic-like equations and Hyperbolic-like equations. Several authors have solved these linear and nonlinear equations using several methods, for example (ADM), (HPM), (VIM), (HAM) ( [2], [3], [6],[7], [8], [9], [10], [11], and [17]). In this article, we are concerned with the following problems:

$$
\begin{cases}u_{t}+f_{1}(x, y, z) u_{x x}+f_{2}(x, y, z) u_{y y}+f_{3}(x, y, z) u_{z z}=0, & t \succ 0 \\ u(x, y, z, 0)=f_{4}(x, y, z), & t=0\end{cases}
$$

and

$$
\left\{\begin{array}{l}
u_{t t}+g_{1}(x, y, z) u_{x x}+g_{2}(x, y, z) u_{y y}+g_{3}(x, y, z) u_{z z}=0, \quad t \succ 0 \\
u(x, y, z, 0)=g_{4}(x, y, z), u_{t}(x, y, z, 0)=g_{5}(x, y, z), \quad t=0 .
\end{array}\right.
$$

For solving these equations, we used the homotopy perturbation transform method (HPTM).

These problems have been studied by some researchers by using (ADM) and (HPM) see for example [12]and [18]. We extend our study to the fractional order for solving the problems:

$$
\left\{\begin{array}{l}
D_{* t}^{\alpha} u(x, y, z, t)+f_{1}(x, y, z) u_{x x}+f_{2}(x, y, z) u_{y y}+f_{3}(x, y, z) u_{z z}=0, t \succ 0 \\
0 \prec \alpha \leq 1, \quad u(x, y, z, 0)=f_{4}(x, y, z), \quad t=0,
\end{array}\right.
$$

and

$$
\left\{\begin{array}{l}
D_{* t}^{\alpha} u(x, y, z, t)+g_{1}(x, y, z) u_{x x}+g_{2}(x, y, z) u_{y y}+g_{3}(x, y, z) u_{z z}=0, t \succ 0 \\
1 \prec \alpha \leq 2, \quad u(x, y, z, 0)=g_{4}(x, y, z), \quad u_{t}(x, y, z, 0)=g_{5}(x, y, z), \quad t=0 .
\end{array}\right.
$$

When we take $\alpha=1(\alpha=2)$ in the equation (3) ((4)), we obtain the exact solution of equation (1) ((2)) successively. Our aim is to solve these problems by using (HPTM) (see [13]).

AbdelkaderKehaili, Laboratory ACEDP, DjillaliLiabes University, 22000 SIDI-BEL-ABBES, Algeria.

Ali Hakem, Laboratory ACEDP, DjillaliLiabes University, 22000 SIDI-BEL-ABBES, Algeria.

AbdelkaderBenali, Faculty of the Exact Sciences and Computer, Mathematics Department, University of Hassiba Benbouali, Chlef Algeria. B. P. 151 Hay Essalem, Chlef 02000, Algeria. 


\section{Basic definitions:}

Definition 2.1. [16] Let $f(t)$ be a function of $t$ specified for $t \succ 0$. Then the Laplace transform of $f(t)$, denoted by $L\{f(t)\}$, and is defined by

$L\{f(t)\}=F(s)=\int_{0}^{\infty} e^{-s t} f(t) d t$,

where we assume at present that the parameter $s$ is strictly positive real number.

Theorem 2.2. [16] If $c_{1}$ and $c_{2}$ are any constants while $f_{1}(t)$ and $f_{2}(t)$ are functions with Laplace transforms $F_{1}(s)$ and $F_{2}(s)$ respectively, then

$L\left\{c_{1} f_{1}(t)+c_{2} f_{2}(t)\right\}=c_{1} L\left\{f_{1}(t)\right\}+c_{2} L\left\{f_{2}(t)\right\}=c_{1} F_{1}(s)+c_{2} F_{2}(s)$.

Theorem 2.3. [15] If

$f(t)=\sum_{n=0}^{\infty} a_{n} t^{n}$

Converge for $t \geq 0$, with

$\left|a_{n}\right| \leq \frac{K \alpha^{n}}{n !}$

for all $n$ sufficiently large and $\alpha \succ 0, K \succ 0$, then

$L\{f(t)\}=\sum_{n=0}^{\infty} a_{n} L\left\{t^{n}\right\}=\sum_{n=0}^{+\infty} \frac{a_{n} n !}{s^{n+1}} \quad(\operatorname{Re}(s) \succ \alpha)$.

Theorem 2.4. [15] Suppose that $f(t), f^{\prime}(t), \ldots, f^{(n-1)}(t)$ are continuous on $[0, \infty)$ and of exponential order, while $f^{n}(t)$ is piecewise continuous on $[0, \infty)$. Then

$$
L\left\{f^{n}(t)\right\}=s^{n} L\{f(t)\}-s^{n-1} f\left(0^{+}\right)-s^{n-2} f^{\prime}\left(0^{+}\right)-\ldots-f^{n-1}\left(0^{+}\right) .
$$

Definition 2.5. [19] A real function $f(t), t \succ 0$, is said to be in the space $C_{\mu}, \mu \in \square$ if there exists a real number $p \succ \mu$, such that $f(t)=t^{p} f_{1}(t)$, where $f_{1}(t) \in C([0, \infty))$, and it is said to be in the space $C_{\mu}^{m}, m \in \square$ if $f^{(m)} \in C_{\mu}$.

Definition 2.6. [19] The fractional derivative of $f \in C_{-1}^{m}$ in the Caputo's sense is defined as:

$$
D_{*}^{\alpha} f(t)=\left\{\begin{array}{l}
\frac{1}{\Gamma(m-\alpha)} \int_{0}^{t}(t-\tau)^{m-\alpha-1} f^{(m)}(\tau) d \tau, \quad \text { if } \quad m-1 \prec \alpha \prec m \\
\frac{d^{m}}{d t^{m}} f(t), \quad \text { if } \quad \alpha=m,
\end{array}\right.
$$

where $m \in \square^{*}$.

Definition 2.7. [19] The Laplace transform, $L\left\{D_{*}^{\alpha} f(t) ; s\right\}$ of the Caputo's fractional derivative is defined as:

$L\left\{D_{*}^{\alpha} f(t) ; s\right\}=s^{\alpha} F(s)-\sum_{k=0}^{m-1} s^{\alpha-k-1} f^{(k)}(0)$,

where $m-1 \prec \alpha \prec m, m \in \square^{*}$.

Analysis of Homotopy perturbation transform method (HPTM):

To illustrate the basic idea of (HPTM) [13], we consider a general nonlinear partial differential equation with the initial conditions of the form $\left\{\begin{array}{l}D u(x, t)+R u(x, t)+N u(x, t)=g(x, t) \\ u(x, 0)=h(x), \quad u_{t}(x, 0)=f(x),\end{array}\right.$

where $D$ in the second order linear differential operator $D=\partial^{2} / \partial t^{2}, R$ is the linear differential operator of less order than $D, N$ represents the general nonlinear differential operator and $g(x, t)$ is the source term. Taking the Laplace transform on both sides of Eq. (12):

$L\{D u(x, t)\}+L\{R u(x, t)\}+L\{N u(x, t)\}=L\{g(x, t)\}$. 
Using the differentiation property of the Laplace transform, we have

$$
L\{u(x, t)\}=\frac{h(x)}{s}+\frac{f(x)}{s^{2}}-\frac{1}{s^{2}} L\{R u(x, t)\}-\frac{1}{s^{2}} L\{N u(x, t)\}+\frac{1}{s^{2}} L\{g(x, t)\} \text {. }
$$

Operating with the Laplace inverse on both sides of Eq. (14) gives

$$
u(x, t)=G(x, t)-L^{-1}\left\{\frac{1}{s^{2}} L\{R u(x, t)+N u(x, t)\}\right\},
$$

where $G(x, t)$ represents the term arising from the source term and the prescribed initial conditions. Now, we apply the homotopy perturbation method

$$
u(x, t)=\sum_{n=0}^{+\infty} p^{n} u_{n}(x, t)
$$

and the nonlinear term can be decomposed as

$$
N u(x, t)=\sum_{n=0}^{+\infty} p^{n} H_{n}(u),
$$

for some He's polynomials $H_{n}(u)$ see ([4]-[5]) that are given by

$$
H_{n}\left(u_{0}, \ldots, u_{n}\right)=\frac{1}{n !} \frac{\partial^{n}}{\partial p^{n}}\left[N\left(\sum_{i=0}^{+\infty} p^{i} u_{i}\right)\right]_{p=0} \quad, n=0,1,2,3, \ldots
$$

Substituting Eqs.(16) and (17) in Eq. (15) we get

$$
\sum_{n=0}^{+\infty} p^{n} u_{n}(x, t)=G(x, t)-p\left(L^{-1}\left\{\frac{1}{s^{2}} L\left\{R \sum_{n=0}^{+\infty} p^{n} u_{n}(x, t)+\sum_{n=0}^{+\infty} p^{n} H_{n}(u)\right\}\right\}\right),
$$

which is the coupling of the Laplace transform and the homotopy perturbation method He's polynomials. Comparing the coefficient of like powers of $p$, the following approximations are obtained

$$
\begin{aligned}
& p^{0}: u_{0}(x, t)=G(x, t), \\
& p^{1}: u_{1}(x, t)=-L^{-1}\left\{\frac{1}{s^{2}} L\left\{R u_{0}(x, t)+H_{0}(u)\right\}\right\}, \\
& p^{2}: u_{2}(x, t)=-L^{-1}\left\{\frac{1}{s^{2}} L\left\{R u_{1}(x, t)+H_{1}(u)\right\}\right\}, \\
& p^{3}: u_{3}(x, t)=-L^{-1}\left\{\frac{1}{s^{2}} L\left\{R u_{2}(x, t)+H_{2}(u)\right\}\right\},
\end{aligned}
$$

Then the solution is

$$
u(x, t)=\lim _{p \rightarrow 1} \sum_{i=0}^{+\infty} p^{i} u_{i}(x, t)=u_{0}(x, t)+u_{1}(x, t)+u_{2}(x, t)+\ldots
$$

\section{Parabolic-like equation:}

Consider the parabolic-like equation in three dimensions of the form:

$$
\left\{\begin{array}{l}
u_{t}+f_{1}(x, y, z) u_{x x}+f_{2}(x, y, z) u_{y y}+f_{3}(x, y, z) u_{z z}=0, \quad t \succ 0 \\
u(x, y, z, 0)=f_{4}(x, y, z), \quad t=0 .
\end{array}\right.
$$

Taking the Laplace transform on both sides of Eq. (21), we get:

$$
L\left\{u_{t}\right\}=-L\left\{f_{1}(x, y, z) u_{x x}+f_{2}(x, y, z) u_{y y}+f_{3}(x, y, z) u_{z z}\right\} \text {. }
$$

An application of Eq. (10), yields:

$L\{u(x, y, z, t)\}=s^{-1} f_{4}(x, y, z)-s^{-1} L\left\{f_{1}(x, y, z) u_{x x}+f_{2}(x, y, z) u_{y y}+f_{3}(x, y, z) u_{z z}\right\}$.

Applying the inverse Laplace transform on both sides in Eq. (23), we get:

$u(x, y, z, t)=f_{4}(x, y, z)-L^{-1}\left\{s^{-1} L\left\{f_{1}(x, y, z) u_{x x}+f_{2}(x, y, z) u_{y y}+f_{3}(x, y, z) u_{z z}\right\}\right\}$.

Applying the classical perturbation technique, we can assume that the solution can be expressed as a power series in $p$, as given below:

$u(x, y, z, t)=\sum_{n=0}^{+\infty} p^{n} u_{n}(x, y, z, t)$, 
where the homotopy parameter $p$, is considered as a small parameter $p \in[0,1]$.

Substituting Eq. (25) in Eq. (24), we get:

$\sum_{n=0}^{+\infty} p^{n} u_{n}=f_{4}-p L^{-1}\left\{s^{-1} L\left\{f_{1} \sum_{n=0}^{+\infty} p^{n}\left(u_{n}\right)_{x x}+f_{2} \sum_{n=0}^{+\infty} p^{n}\left(u_{n}\right)_{y y}+f_{3} \sum_{n=0}^{+\infty} p^{n}\left(u_{n}\right)_{z z}\right\}\right\}$,

this is a coupling of the Laplace transform and homotopy perturbation methods using He's polynomials. Now, equating the coefficient of corresponding power of $\mathrm{p}$ on both sides, the following approximations are obtained as:

$$
\begin{aligned}
& p^{0}: u_{0}(x, y, z, t)=f_{4}(x, y, z), \\
& p^{1}: u_{1}(x, y, z, t)=-L^{-1}\left\{s^{-1} L\left\{f_{1}\left(u_{0}\right)_{x x}+f_{2}\left(u_{0}\right)_{y y}+f_{3}\left(u_{0}\right)_{z z}\right\}\right\}, \\
& \vdots \\
& p^{n}: u_{n}(x, y, z, t)=-L^{-1}\left\{s^{-1} L\left\{f_{1}\left(u_{n-1}\right)_{x x}+f_{2}\left(u_{n-1}\right)_{y y}+f_{3}\left(u_{n-1}\right)_{z z}\right\}\right\},
\end{aligned}
$$

where $n \in \square^{*}$.

Proceeding in this same manner, the rest of the components $u_{n}(x, y, z, t)$, can be completely obtained, and the series solution is thus entirely determined. Finally, we approximate the analytical solution $u(x, y, z, t)$, by truncated series:

$u(x, y, z, t)=\lim _{N \rightarrow \infty} \sum_{n=0}^{N} u_{n}(x, y, z, t)$.

\section{Hyperbolic-like equation:}

Consider the three dimensional hyperbolic-like equation of the form:

$$
\left\{\begin{array}{l}
u_{t t}+g_{1}(x, y, z) u_{x x}+g_{2}(x, y, z) u_{y y}+g_{3}(x, y, z) u_{z z}=0, \quad t \succ 0 \\
u(x, y, z, 0)=g_{4}(x, y, z), u_{t}(x, y, z, 0)=g_{5}(x, y, z), \quad t=0 .
\end{array}\right.
$$

Taking the Laplace transform on both sides of Eq. (28), we get:

$$
L\left\{u_{t t}\right\}=-L\left\{g_{1}(x, y, z) u_{x x}+g_{2}(x, y, z) u_{y y}+g_{3}(x, y, z) u_{z z}\right\} \text {. }
$$

An application of Eq. (10), yields:

$$
L\{u\}=s^{-1} g_{4}+s^{-2} g_{5}-s^{-2} L\left\{g_{1}(x, y, z) u_{x x}+g_{2}(x, y, z) u_{y y}+g_{3}(x, y, z) u_{z z}\right\} \text {. }
$$

Applying the inverse Laplace transform on both sides in Eq. (30), we get:

$$
u(x, y, z, t)=g_{4}+\operatorname{tg} g_{5}-L^{-1}\left\{s^{-2} L\left\{g_{1} u_{x x}+g_{2} u_{y y}+g_{3} u_{z z}\right\}\right\} \text {. }
$$

Now applying the classical perturbation technique, we can assume that the solution can be expressedas a power series in $p$, as given below:

$$
u(x, y, z, t)=\sum_{n=0}^{+\infty} p^{n} u_{n}(x, y, z, t),
$$

where the homotopy parameter $\mathrm{p}$, is considered as a small parameter $p \in[0,1]$.

Substituting Eq. (32) in Eq. (31), we get:

$$
\sum_{n=0}^{+\infty} p^{n} u_{n}=g_{4}+\operatorname{tg}_{5}-p L^{-1}\left\{s^{-2} L\left\{g_{1} \sum_{n=0}^{+\infty} p^{n}\left(u_{n}\right)_{x x}+g_{2} \sum_{n=0}^{+\infty} p^{n}\left(u_{n}\right)_{y y}+g_{3} \sum_{n=0}^{+\infty} p^{n}\left(u_{n}\right)_{z z}\right\}\right\},
$$

this is a coupling of the Laplace transform and homotopy perturbation methods using He's polynomials. Now, equating the coefficient of corresponding power of $p$ on both sides, the following approximations are obtained as:

$$
\begin{aligned}
& p^{0}: u_{0}(x, y, z, t)=g_{4}+\operatorname{tg}_{5}, \\
& p^{1}: u_{1}(x, y, z, t)=-L^{-1}\left\{s^{-2} L\left\{g_{1}\left(u_{0}\right)_{x x}+g_{2}\left(u_{0}\right)_{y y}+g_{3}\left(u_{0}\right)_{z z}\right\}\right\}, \\
& \vdots \\
& p^{n}: u_{n}(x, y, z, t)=-L^{-1}\left\{s^{-2} L\left\{g_{1}\left(u_{n-1}\right)_{x x}+g_{2}\left(u_{n-1}\right)_{y y}+g_{3}\left(u_{n-1}\right)_{z z}\right\}\right\},
\end{aligned}
$$

where $n \in \square$ *. 
Proceeding in this same manner, the rest of the components $u_{n}(x, y, z, t)$, can be completely obtained, and the series solution is thus entirely determined. Finally, we approximate the analytical solution $u(x, y, z, t)$, by truncated series:

$u(x, y, z, t)=\lim _{N \rightarrow \infty} \sum_{n=0}^{N} u_{n}(x, y, z, t)$.

\section{Numerical examples:}

Example 6.1. : Consider the following one-dimensional parabolic-like equation with variable coefficients:

$$
\left\{\begin{array}{l}
u_{t}(x, t)-\left(\frac{1}{2} x^{2}-\frac{1}{4}\right) u_{x x}=0, \quad t \succ 0 \\
u(x, 0)=2 x^{2}-1, \quad t=0 .
\end{array}\right.
$$

Taking the Laplace transform on both sides of Eq. (35), we get:

$$
L\left\{u_{t}(x, t)\right\}=L\left\{\left(\frac{1}{2} x^{2}-\frac{1}{4}\right) u_{x x}\right\} .
$$

An application of Eq. (10), yields:

$$
L\{u(x, t)\}=s^{-1}\left(2 x^{2}-1\right)+s^{-1} L\left\{\left(\frac{1}{2} x^{2}-\frac{1}{4}\right) u_{x x}\right\} .
$$

Applying the inverse Laplace transform on both sides in Eq. (37), we get:

$u(x, t)=\left(2 x^{2}-1\right)+L^{-1}\left\{s^{-1} L\left\{\left(\frac{1}{2} x^{2}-\frac{1}{4}\right) u_{x x}\right\}\right\}$.

By applying the aforesaid homotopy perturbation method, we have:

$\sum_{n=0}^{+\infty} p^{n} u_{n}=2 x^{2}-1+p L^{-1}\left\{s^{-1} L\left\{\left(\frac{1}{2} x^{2}-\frac{1}{4}\right) \sum_{n=0}^{+\infty} p^{n}\left(u_{n}\right)_{x x}(x, t)\right\}\right\}$.

Equating the coefficient of the like power of $p$ on both sides in Eq. (39), we get:

$$
\begin{aligned}
& p^{0}: u_{0}(x, t)=2 x^{2}-1, \\
& p^{1}: u_{1}(x, t)=L^{-1}\left\{s^{-1} L\left\{\left(\frac{1}{2} x^{2}-\frac{1}{4}\right)\left(u_{0}\right)_{x x}\right\}\right\}, \\
& \vdots \\
& p^{n}: u_{n}(x, t)=L^{-1}\left\{s^{-1} L\left\{\left(\frac{1}{2} x^{2}-\frac{1}{4}\right)\left(u_{n-1}\right)_{x x}\right\}\right\},
\end{aligned}
$$

where $n \in \square^{*}$.

Using the iteration formula (40), we obtain

$$
\begin{aligned}
& u_{0}(x, t)=2 x^{2}-1, \\
& u_{1}(x, t)=\left(2 x^{2}-1\right) t, \\
& u_{2}(x, t)=\left(2 x^{2}-1\right) \frac{t^{2}}{2 !}, \\
& \vdots \\
& u_{n}(x, t)=\left(2 x^{2}-1\right) \frac{t^{n}}{n !} .
\end{aligned}
$$

Finally, we approximate the analytical solution $u(x, t)$, by truncated series:

$$
u(x, t)=\lim _{N \rightarrow \infty} \sum_{n=0}^{N} u_{n}(x, t)=\left(2 x^{2}-1\right) e^{t} .
$$


Example 6.2. : Consider the following two-dimensional parabolic-like equation with variable coefficients:

$$
\left\{\begin{array}{l}
u_{t}(x, y, t)+\frac{y^{2}-1}{2} u_{x x}-\frac{x^{2}+1}{2} u_{y y}=0, \quad t \succ 0 \\
u(x, y, 0)=y^{2}-1, \quad t=0 .
\end{array}\right.
$$

Taking the Laplace transform on both sides of Eq. (42), we get:

$L\left\{u_{t}(x, y, t)\right\}=L\left\{\frac{x^{2}+1}{2} u_{y y}-\frac{y^{2}-1}{2} u_{x x}\right\}$.

An application of Eq. (10), yields:

$L\{u(x, y, t)\}=s^{-1}\left(y^{2}-1\right)+s^{-1} L\left\{\frac{x^{2}+1}{2} u_{y y}-\frac{y^{2}-1}{2} u_{x x}\right\}$.

Applying the inverse Laplace transform on both sides in Eq. (44), we get:

$u(x, y, t)=y^{2}-1+L^{-1}\left\{s^{-1} L\left\{\frac{x^{2}+1}{2} u_{y y}-\frac{y^{2}-1}{2} u_{x x}\right\}\right\}$.

By applying the aforesaid homotopy perturbation method, we have:

$\sum_{n=0}^{+\infty} p^{n} u_{n}=y^{2}-1+p L^{-1}\left\{s^{-1} L\left\{\frac{x^{2}+1}{2} \sum_{n=0}^{+\infty} p^{n}\left(u_{n}\right)_{Y Y}-\frac{y^{2}-1}{2} \sum_{n=0}^{+\infty} p^{n}\left(u_{n}\right)_{x x}\right\}\right\}$.

Equating the coefficient of the like power of $p$ on both sides in Eq. (46), we get

$p^{0}: u_{0}(x, y, t)=y^{2}-1$,

$p^{1}: u_{1}(x, y, t)=L^{-1}\left\{s^{-1} L\left\{\frac{x^{2}+1}{2}\left(u_{0}\right)_{y y}-\frac{y^{2}-1}{2}\left(u_{0}\right)_{x x}\right\}\right\}$,

$\vdots$

$p^{n}: u_{n}(x, y, t)=L^{-1}\left\{s^{-1} L\left\{\frac{x^{2}+1}{2}\left(u_{n-1}\right)_{y y}-\frac{y^{2}-1}{2}\left(u_{n-1}\right)_{x x}\right\}\right\}$,

where $n \in \square^{*}$.

Using the iteration formula (47), we obtain

$u_{0}(x, y, t)=y^{2}-1$,

$u_{1}(x, y, t)=\left(x^{2}+1\right) t$,

$u_{2}(x, y, t)=-\left(y^{2}-1\right) \frac{t^{2}}{2 !}$,

$u_{3}(x, y, t)=-\left(x^{2}+1\right) \frac{t^{3}}{3 !}$

$\vdots$

$u_{2 n}(x, y, t)=(-1)^{n}\left(y^{2}-1\right) \frac{t^{2 n}}{(2 n) !}$,

$u_{2 n+1}(x, y, t)=(-1)^{n}\left(x^{2}+1\right) \frac{t^{2 n+1}}{(2 n+1) !}$.

Finally, we approximate the analytical solution $u(x, y, t)$, by truncated series:

$u(x, y, t)=\lim _{N \rightarrow \infty} \sum_{n=0}^{N} u_{n}(x, y, t)=\left(x^{2}+1\right) \sin t+\left(y^{2}-1\right) \cos t$. 
Example 6.3. : Consider the following three-dimensional parabolic-like equation with variable coefficients:

$\left\{\begin{array}{lr}u_{t}(x, y, z, t)=\frac{x^{2}}{12} u_{x x}+\frac{y^{2}}{24} u_{y y}+\frac{z^{2}}{24} u_{z z}, \quad t \succ 0 \\ u(x, y, z, 0)=2 x^{3} y^{3} z^{3}, \quad t=0 . & \end{array}\right.$

Taking the Laplace transform on both sides of Eq. (49), we get:

$$
L\left\{u_{t}(x, y, z, t)\right\}=L\left\{\frac{x^{2}}{12} u_{x x}+\frac{y^{2}}{24} u_{y y}+\frac{z^{2}}{24} u_{z z}\right\} .
$$

An application of Eq. (10), yields:

$L\{u(x, y, z, t)\}=s^{-1}\left(2 x^{3} y^{3} z^{3}\right)+s^{-1} L\left\{\frac{x^{2}}{12} u_{x x}+\frac{y^{2}}{24} u_{y y}+\frac{z^{2}}{24} u_{z z}\right\}$.

Applying the inverse Laplace transform on both sides in Eq. (51), we get:

$u(x, y, z, t)=2 x^{3} y^{3} z^{3}+L^{-1}\left\{s^{-1} L\left\{\frac{x^{2}}{12} u_{x x}+\frac{y^{2}}{24} u_{y y}+\frac{z^{2}}{24} u_{z z}\right\}\right\}$.

By applying the aforesaid homotopy perturbation method, we have:

$\sum_{n=0}^{+\infty} p^{n} u_{n}=2 x^{3} y^{3} z^{3}+p L^{-1}\left\{s^{-1} L\left\{\frac{x^{2}}{12} \sum_{n=0}^{+\infty} p^{n}\left(u_{n}\right)_{x x}+\frac{y^{2}}{24} \sum_{n=0}^{+\infty} p^{n}\left(u_{n}\right)_{y y}+\frac{z^{2}}{24} \sum_{n=0}^{+\infty} p^{n}\left(u_{n}\right)_{z z}\right\}\right\}$.

Equating the coefficient of the like power of $p$ on both sides in Eq. (53), weget :

$p^{0}: u_{0}(x, y, z, t)=2 x^{3} y^{3} z^{3}$,

$p^{1}: u_{1}(x, y, z, t)=L^{-1}\left\{s^{-1} L\left\{\frac{x^{2}}{12}\left(u_{0}\right)_{x x}+\frac{y^{2}}{24}\left(u_{0}\right)_{y y}+\frac{z^{2}}{24}\left(u_{0}\right)_{z z}\right\}\right\}$,

$\vdots$

$p^{n}: u_{n}(x, y, z, t)=L^{-1}\left\{s^{-1} L\left\{\frac{x^{2}}{12}\left(u_{n-1}\right)_{x x}+\frac{y^{2}}{24}\left(u_{n-1}\right)_{y y}+\frac{z^{2}}{24}\left(u_{n-1}\right)_{z z}\right\}\right\}$,

where $n \in \square^{*}$.

Using the iteration formula (54), we obtain

$u_{0}(x, y, z, t)=2 x^{3} y^{3} z^{3}$,

$u_{1}(x, y, z, t)=2 x^{3} y^{3} z^{3} t$,

$u_{2}(x, y, z, t)=2 x^{3} y^{3} z^{3} \frac{t^{2}}{2 !}$,

$\vdots$

$u_{n}(x, y, z, t)=2 x^{3} y^{3} z^{3} \frac{t^{n}}{n !}$.

Finally, we approximate the analytical solution $u(x, y, z, t)$, by truncated series:

$u(x, y, z, t)=\lim _{N \rightarrow \infty} \sum_{n=0}^{N} u_{n}(x, y, z, t)=2 x^{3} y^{3} z^{3} e^{t}$.

Example 6.4. : Consider the following one-dimensional hyperbolic-like equation with variable coefficients:

$\left\{\begin{array}{l}u_{t t}(x, t)=\frac{x^{2}-1}{2} u_{x x}, \quad t \succ 0 \\ u(x, 0)=x^{2}-1, \quad u_{t}(x, 0)=0 \quad t=0 .\end{array}\right.$ 
Taking the Laplace transform on both sides of Eq. (56), we get:

$L\left\{u_{t t}(x, t)\right\}=L\left\{\frac{x^{2}-1}{2} u_{x x}\right\}$.

An application of $(10)$ yields:

$L\{u(x, t)\}=s^{-1}\left(x^{2}-1\right)+s^{-2} L\left\{\frac{x^{2}-1}{2} u_{x x}\right\}$.

Applying the inverse Laplace transform on both sides of Eq. (58), we get:

$u(x, t)=x^{2}-1+L^{-1}\left\{s^{-2} L\left\{\frac{x^{2}-1}{2} u_{x x}\right\}\right\}$.

By applying the aforesaid homotopy perturbation method, we have:

$\sum_{n=0}^{+\infty} p^{n} u_{n}=x^{2}-1+p L^{-1}\left\{s^{-2} L\left\{\frac{x^{2}-1}{2} \sum_{n=0}^{+\infty} p^{n}\left(u_{n}\right)_{x x}(x, t)\right\}\right\}$.

Equating the coefficient of the like power of $\mathrm{p}$ on both sides in Eq. (60), we get:

$p^{0}: u_{0}(x, t)=x^{2}-1$,

$p^{1}: u_{1}(x, t)=L^{-1}\left\{s^{-2} L\left\{\frac{x^{2}-1}{2}\left(u_{0}\right)_{x x}\right\}\right\}$,

$\vdots$

$p^{n}: u_{n}(x, t)=L^{-1}\left\{s^{-2} L\left\{\frac{x^{2}-1}{2}\left(u_{n-1}\right)_{x x}\right\}\right\}$,

where $n \in \square^{*}$.

Using the iteration formula (61), we obtain

$u_{0}(x, t)=x^{2}-1$,

$u_{1}(x, t)=\left(x^{2}-1\right) \frac{t^{2}}{2 !}$,

$\vdots$

$u_{n}(x, t)=\left(x^{2}-1\right) \frac{t^{2 n}}{(2 n) !}$.

Finally, we approximate the analytical solution $u(x, t)$, by truncated series:

$u(x, t)=\lim _{N \rightarrow \infty} \sum_{n=0}^{N} u_{n}(x, t)=\left(x^{2}-1\right) \cosh t$.

Example 6.5. : Consider the following two-dimensional hyperbolic-like equation with variable coefficients:

$\left\{\begin{array}{l}u_{t t}(x, y, t)=\frac{1}{6} x^{2} u_{x x}+\frac{1}{6} y^{2} u_{y y}, \quad t \succ 0 \\ u(x, y, 0)=0, \quad u_{t}(x, y, 0)=x^{3}+y^{3}, \quad t=0 .\end{array}\right.$

Taking the Laplace transform on both sides of Eq. (63), we get:

$$
L\left\{u_{t t}(x, y, t)\right\}=L\left\{\frac{1}{6} x^{2} u_{x x}+\frac{1}{6} y^{2} u_{y y}\right\} .
$$

An application of Eq. (10), yields:

$$
L\{u(x, y, t)\}=s^{-2}\left(x^{3}+y^{3}\right)+s^{-2} L\left\{\frac{1}{6} x^{2} u_{x x}+\frac{1}{6} y^{2} u_{y y}\right\} \text {. }
$$


Applying the inverse Laplace transform on both sides in Eq. (65), we get:

$u(x, y, t)=\left(x^{3}+y^{3}\right) t+L^{-1}\left\{s^{-2} L\left\{\frac{1}{6} x^{2} u_{x x}+\frac{1}{6} y^{2} u_{y y}\right\}\right\}$.

By applying the aforesaid homotopy perturbation method, we have:

$\sum_{n=0}^{+\infty} p^{n} u_{n}=\left(x^{3}+y^{3}\right) t+p L^{-1}\left\{s^{-2} L\left\{\frac{1}{6} x^{2} \sum_{n=0}^{+\infty} p^{n}\left(u_{n}\right)_{x x}+\frac{1}{6} y^{2} \sum_{n=0}^{+\infty} p^{n}\left(u_{n}\right)_{Y Y}\right\}\right\}$.

Equating the coefficient of the like power of $p$ on both sides in Eq. (67), we get:

$p^{0}: u_{0}(x, y, t)=\left(x^{3}+y^{3}\right) t$,

$p^{1}: u_{1}(x, y, t)=L^{-1}\left\{s^{-2} L\left\{\frac{1}{6} x^{2}\left(u_{0}\right)_{x x}+\frac{1}{6} y^{2}\left(u_{0}\right)_{y y}\right\}\right\}$,

$p^{n}: u_{n}(x, y, t)=L^{-1}\left\{s^{-2} L\left\{\frac{1}{6} x^{2}\left(u_{n-1}\right)_{x x}+\frac{1}{6} y^{2}\left(u_{n-1}\right)_{y y}\right\}\right\}$,

where $n \in \square^{*}$.

Using the iteration formula (68), we obtain

$$
\begin{aligned}
& u_{0}(x, y, t)=\left(x^{3}+y^{3}\right) t, \\
& u_{1}(x, y, t)=\left(x^{3}+y^{3}\right) \frac{t^{3}}{3 !}, \\
& \vdots \\
& u_{n}(x, y, t)=\left(x^{3}+y^{3}\right) \frac{t^{2 n+1}}{(2 n+1) !} .
\end{aligned}
$$

Finally, we approximate the analytical solution $u(x, y, t)$, by truncated series:

$$
u(x, y, t)=\lim _{N \rightarrow \infty} \sum_{n=0}^{N} u_{n}(x, y, t)=\left(x^{3}+y^{3}\right) \sinh t \text {. }
$$

Example 6.6. : Consider the following three-dimensional hyperbolic-like equation with variable coefficients:

$$
\begin{cases}u_{t t}(x, y, z, t)=-\frac{1}{2} x^{2} u_{x x}-\frac{1}{2} y^{2} u_{y y}-\frac{1}{2} z^{2} u_{z z}, \quad t \succ 0 \\ u(x, y, z, 0)=0, & u_{t}(x, y, z, 0)=x^{2}+2 y^{2}+3 z^{2}, \quad t=0 .\end{cases}
$$

Taking the Laplace transform on both sides of Eq. (70), we get:

$$
L\left\{u_{t t}(x, y, z, t)\right\}=L\left\{-\frac{1}{2} x^{2} u_{x x}-\frac{1}{2} y^{2} u_{y y}-\frac{1}{2} z^{2} u_{z z}\right\} \text {. }
$$

An application of Eq. (10), yields:

$$
L\{u(x, y, z, t)\}=s^{-2}\left(x^{2}+2 y^{2}+3 z^{2}\right)+s^{-2} L\left\{-\frac{1}{2} x^{2} u_{x x}-\frac{1}{2} y^{2} u_{y y}-\frac{1}{2} z^{2} u_{z z}\right\} \text {. }
$$

Applying the inverse Laplace transform on both sides in Eq. (72), we get:

$$
u(x, y, z, t)=\left(x^{2}+2 y^{2}+3 z^{2}\right) t+L^{-1}\left\{s^{-2} L\left\{-\frac{1}{2} x^{2} u_{x x}-\frac{1}{2} y^{2} u_{y y}-\frac{1}{2} z^{2} u_{z z}\right\}\right\} \text {. }
$$

By applying the aforesaid homotopy perturbation method, we have:

$$
\sum_{n=0}^{+\infty} p^{n} u_{n}=\left(x^{2}+2 y^{2}+3 z^{2}\right) t+p L^{-1}\left\{s^{-2} L\left\{-\frac{1}{2} x^{2} \sum_{n=0}^{+\infty} p^{n}\left(u_{n}\right)_{x x}-\frac{1}{2} y^{2} \sum_{n=0}^{+\infty} p^{n}\left(u_{n}\right)_{y y}-\frac{1}{2} z^{2} \sum_{n=0}^{+\infty} p^{n}\left(u_{n}\right)_{z z}\right\}\right\} \text {. (74 }
$$


Equating the coefficient of the like power of $p$ on both sides in Eq. (74), we get:

$$
\begin{aligned}
& p^{0}: u_{0}(x, y, t)=\left(x^{2}+2 y^{2}+3 z^{2}\right) t, \\
& p^{1}: u_{1}(x, y, t)=L^{-1}\left\{s^{-2} L\left\{-\frac{1}{2} x^{2}\left(u_{0}\right)_{x x}-\frac{1}{2} y^{2}\left(u_{0}\right)_{y y}-\frac{1}{2} z^{2}\left(u_{0}\right)_{z z}\right\}\right\}, \\
& \vdots \\
& p^{n}: u_{n}(x, y, t)=L^{-1}\left\{s^{-2} L\left\{-\frac{1}{2} x^{2}\left(u_{n-1}\right)_{x x}-\frac{1}{2} y^{2}\left(u_{n-1}\right)_{y y}-\frac{1}{2} z^{2}\left(u_{n-1}\right)_{z z}\right\}\right\},
\end{aligned}
$$

where $n \in \square^{*}$.

Using the iteration formula (75), we obtain

$$
\begin{aligned}
& u_{0}(x, y, z, t)=\left(x^{2}+2 y^{2}+3 z^{2}\right) t \\
& u_{1}(x, y, z, t)=\left(x^{2}+2 y^{2}+3 z^{2}\right) \frac{t^{3}}{3 !} \\
& \vdots \\
& u_{n}(x, y, z, t)=(-1)^{n}\left(x^{2}+2 y^{2}+3 z^{2}\right) \frac{t^{2 n+1}}{(2 n+1) !} .
\end{aligned}
$$

Finally, we approximate the analytical solution $u(x, y, z, t)$, by truncated series:

$$
u(x, y, z, t)=\lim _{N \rightarrow \infty} \sum_{n=0}^{N} u_{n}(x, y, z, t)=\left(x^{2}+2 y^{2}+3 z^{2}\right) \sin t
$$

\section{Fractional homotopy perturbation transform method:}

In order to elucidate the solution procedure of the fractional Laplace homotopy perturbation method [14], we consider the following nonlinear fractional differential equation:

$$
\left\{\begin{array}{l}
D_{* t}^{\alpha} u(x, t)+R[x] u(x, t)+N[x] u(x, t)=q(x, t), \quad t \succ 0 \\
0 \prec \alpha \leq 1, \quad u(x, 0)=h(x), \quad t=0,
\end{array}\right.
$$

where $D^{\alpha}=\frac{\partial^{\alpha}}{\partial t^{\alpha}}, R[x]$ is the linear operator in $x, N[x]$ is the general nonlinear operator in $x$, and $q(x, t)$ are continuous functions. Now, the methodology consists of applying the Laplace transform first on both sides of (77). Thus, we get:

$$
L\left\{D_{* t}^{\alpha} u(x, t)\right\}+L\{R[x] u(x, t)+N[x] u(x, t)\}=L\{q(x, t)\} .
$$

Now, using the differentiation property of the Laplace transform, we have:

$$
L\{u(x, t)\}=s^{-1} h(x)+s^{-1} q(x, t)-s^{-\alpha} L\{R[x] u(x, t)+N[x] u(x, t)\} \text {. }
$$

Operating the inverse Laplace transform on both sides in (79), we get:

$$
u(x, t)=G(x, t)-L^{-1}\left\{s^{-\alpha} L\{R[x] u(x, t)+N[x] u(x, t)\}\right\},
$$

where $G(x, t)$, represents the term arising from the source term and the prescribed initial conditions. Now, applying the classical perturbation technique, we can assume that the solution can be expressed as a power series in $p$, as given below:

$$
u(x, t)=\sum_{n=0}^{+\infty} p^{n} u_{n}(x, t),
$$

where the homotopy parameter, $\mathrm{p}$, is considered as a small parameter $p \in[0,1]$. The nonlinear term can be decomposed as:

$$
N u(x, t)=\sum_{n=0}^{+\infty} p^{n} H_{n}(u) \text {, }
$$


where $H_{n}$ are He's polynomials of $u_{0}, u_{1}, u_{2}, \ldots, u_{n}$, which can be calculated by the following formula:

$H_{n}\left(u_{0}, \ldots, u_{n}\right)=\frac{1}{n !} \frac{\partial^{n}}{\partial p^{n}}\left[N\left(\sum_{i=0}^{+\infty} p^{i} u_{i}\right)\right]_{p=0}, n=0,1,2,3, \ldots$

Substituting Eqs. (81) and (82) in Eq. (80) and using HPM by He see ([7]-[8]), we get:

$\sum_{n=0}^{+\infty} p^{n} u_{n}(x, t)=G(x, t)-p\left(L^{-1}\left\{s^{-\alpha} L\left\{R \sum_{n=0}^{+\infty} p^{n} u_{n}(x, t)+\sum_{n=0}^{+\infty} p^{n} H_{n}(u)\right\}\right\}\right)$,

This is a coupling of the Laplace transform and homotopy perturbation methods using He's polynomials. Now, equating the coefficient of corresponding power of $p$ on both sides, the following approximations are obtained as:

$p^{0}: u_{0}(x, t)=G(x, t)$,

$p^{1}: u_{1}(x, t)=-L^{-1}\left\{s^{-\alpha} L\left\{R u_{0}(x, t)+H_{0}(u)\right\}\right\}$,

$\vdots$

$p^{n}: u_{n}(x, t)=-L^{-1}\left\{s^{-\alpha} L\left\{R u_{n-1}(x, t)+H_{n-1}(u)\right\}\right\}$,

where $n \in \square^{*}$.

Proceeding in this same manner, the rest of the components $u_{n}(x, t)$, can be completely obtained, and the series solution is thus entirely determined. Finally, we approximate the analytical solution $u(x, t)$, by truncated series:

$u(x, t)=\lim _{N \rightarrow \infty} \sum_{n=0}^{N} u_{n}(x, t)$.

The above series solutions generally converge very rapidly. A classical approach of convergence of this type of series is already presented by Abbaoui and Cherruault [1].

The time-fractional equations of the form (3):

Consider the following time-fractional equation with variable coefficients:

$\left\{\begin{array}{l}D_{* t}^{\alpha} u(x, y, z, t)+f_{1}(x, y, z) u_{x x}+f_{2}(x, y, z) u_{y y}+f_{3}(x, y, z) u_{z z}=0, t \succ 0 \\ 0 \prec \alpha \leq 1, \quad u(x, y, z, 0)=f_{4}(x, y, z), \quad t=0 .\end{array}\right.$

Taking the Laplace transform on both sides of Eq. (86), we get:

$$
L\left\{D_{* t}^{\alpha} u(x, y, z, t)\right\}=-L\left\{f_{1}(x, y, z) u_{x x}+f_{2}(x, y, z) u_{y y}+f_{3}(x, y, z) u_{z z}\right\} .
$$

An application of Eq. (11), yields:

$L\{u(x, y, z, t)\}=s^{-1} f_{4}(x, y, z)-s^{-\alpha} L\left\{f_{1}(x, y, z) u_{x x}+f_{2}(x, y, z) u_{y y}+f_{3}(x, y, z) u_{z z}\right\}$.

Applying the inverse Laplace transform on both sides in Eq. (88), we get:

$$
u(x, y, z, t)=f_{4}(x, y, z)-L^{-1}\left\{s^{-\alpha} L\left\{f_{1}(x, y, z) u_{x x}+f_{2}(x, y, z) u_{y y}+f_{3}(x, y, z) u_{z z}\right\}\right\} .
$$

Applying the classical perturbation technique, we can assume that the solution can be expressed as a power series in $p$, as given below:

$u(x, y, z, t)=\sum_{n=0}^{+\infty} p^{n} u_{n}(x, y, z, t)$,

where the homotopy parameter $p$, is considered as a small parameter $p \in[0,1]$.

Substituting Eq. (90) in Eq. (89), we get:

$\sum_{n=0}^{+\infty} p^{n} u_{n}=f_{4}-p L^{-1}\left\{s^{-\alpha} L\left\{f_{1} \sum_{n=0}^{+\infty} p^{n}\left(u_{n}\right)_{x x}+f_{2} \sum_{n=0}^{+\infty} p^{n}\left(u_{n}\right)_{y y}+f_{3} \sum_{n=0}^{+\infty} p^{n}\left(u_{n}\right)_{z z}\right\}\right\}$. 
This is a coupling of the Laplace transform and homotopy perturbation methods using He's polynomials. Now, equating the coefficient of corresponding power of $p$ on both sides, the following approximations are obtained as:

$$
\begin{aligned}
& p^{0}: u_{0}(x, y, z, t)=f_{4}(x, y, z), \\
& \vdots \\
& p^{n}: u_{n}(x, y, z, t)=-L^{-1}\left\{s^{-\alpha} L\left\{f_{1}\left(u_{n-1}\right)_{x x}+f_{2}\left(u_{n-1}\right)_{y y}+f_{3}\left(u_{n-1}\right)_{z z}\right\}\right\},
\end{aligned}
$$

where $n \in \square^{*}$.

Proceeding in this same manner, the rest of the components $u_{n}(x, y, z, t)$, can be completely obtained, and the series solution is thus entirely determined. Finally, we approximate the analytical solution $u(x, y, z, t)$, by truncated series:

$$
u(x, y, z, t)=\lim _{N \rightarrow \infty} \sum_{n=0}^{N} u_{n}(x, y, z, t) .
$$

\section{The time-fractional equations of the form (4):}

Consider the following time-fractional equation with variable coefficients:

$$
\left\{\begin{array}{l}
D_{* t}^{\alpha} u(x, y, z, t)+g_{1}(x, y, z) u_{x x}+g_{2}(x, y, z) u_{y y}+g_{3}(x, y, z) u_{z z}=0, \quad t \succ 0 \\
1 \prec \alpha \leq 2, u(x, y, z, 0)=g_{4}(x, y, z), u_{t}(x, y, z, 0)=g_{5}(x, y, z), t=0 .
\end{array}\right.
$$

Taking the Laplace transform on both sides of Eq. (93), we get:

$$
L\left\{D_{* t}^{\alpha} u(x, y, z, t)\right\}=-L\left\{g_{1}(x, y, z) u_{x x}+g_{2}(x, y, z) u_{y y}+g_{3}(x, y, z) u_{z z}\right\} \text {. }
$$

An application of Eq. (11), yields:

$$
L\{u(x, y, z, t)\}=s^{-1} g_{4}(x, y, z)+s^{-2} g_{5}(x, y, z)-s^{-\alpha} L\left\{g_{1}(x, y, z) u_{x x}+g_{2}(x, y, z) u_{y y}+g_{3}(x, y, z) u_{z z}\right\} \text {. }
$$

Applying the inverse Laplace transform on both sides in Eq. (95), we get:

$$
u(x, y, z)=g_{4}(x, y, z)+\operatorname{tg}_{5}(x, y, z)-L^{-1}\left\{s^{-\alpha} L\left\{g_{1}(x, y, z) u_{x x}+g_{2}(x, y, z) u_{y y}+g_{3}(x, y, z) u_{z z}\right\}\right\} \text {. }
$$

Applying the classical perturbation technique, we can assume that the solution can be expressed as a power series in $p$, as given below:

$$
u(x, y, z, t)=\sum_{n=0}^{+\infty} p^{n} u_{n}(x, y, z, t)
$$

where the homotopy parameter $p$, is considered as a small parameter $p \in[0,1]$.

Substituting Eq. (97) in Eq. (96), we get:

$$
\sum_{n=0}^{+\infty} p^{n} u_{n}=g_{4}+\operatorname{tg}_{5}-p L^{-1}\left\{s^{-\alpha} L\left\{g_{1} \sum_{n=0}^{+\infty} p^{n}\left(u_{n}\right)_{x x}+g_{2} \sum_{n=0}^{+\infty} p^{n}\left(u_{n}\right)_{y y}+g_{3} \sum_{n=0}^{+\infty} p^{n}\left(u_{n}\right)_{z z}\right\}\right\} \text {. }
$$

This is a coupling of the Laplace transform and homotopy perturbation methods using He's polynomials. Now, equating the coefficient of corresponding power of $p$ on both sides, the following approximations are obtained as:

$$
\begin{aligned}
& p^{0}: u_{0}(x, y, z, t)=g_{4}(x, y, z)+\operatorname{tg}_{5}(x, y, z), \\
& \vdots \\
& p^{n}: u_{n}(x, y, z, t)=-L^{-1}\left\{s^{-\alpha} L\left\{g_{1}\left(u_{n-1}\right)_{x x}+g_{2}\left(u_{n-1}\right)_{y y}+g_{3}\left(u_{n-1}\right)_{z z}\right\}\right\},
\end{aligned}
$$

where $n \in \square^{*}$.

Proceeding in this same manner, the rest of the components $u_{n}(x, y, z, t)$, can be completely obtained, and the series solution is thus entirely determined. Finally, we approximate the analytical solution $u(x, y, z, t)$, by truncated series:

$$
u(x, y, z, t)=\lim _{N \rightarrow \infty} \sum_{n=0}^{N} u_{n}(x, y, z, t) .
$$


Numerical examples:

Example 10.1. : Consider the following time-fractional equation with variable coefficients:

$\left\{\begin{array}{c}D_{* t}^{\alpha} u(x, t)-\left(\frac{1}{2} x^{2}-\frac{1}{4}\right) u_{x x}=0, \quad t \succ 0 \\ 0 \prec \alpha \leq 1 \quad u(x, 0)=2 x^{2}-1, \quad t=0 .\end{array}\right.$

Taking the Laplace transform on both sides of Eq. (100), we get:

$L\left\{D_{* t}^{\alpha} u(x, t)\right\}=L\left\{\left(\frac{1}{2} x^{2}-\frac{1}{4}\right) u_{x x}\right\}$.

An application of Eq. (11), yields:

$L\{u(x, t)\}=s^{-1}\left(2 x^{2}-1\right)+s^{-\alpha} L\left\{\left(\frac{1}{2} x^{2}-\frac{1}{4}\right) u_{x x}\right\}$.

Applying the inverse Laplace transform of both sides in Eq. (102), we get:

$u(x, t)=2 x^{2}-1+L^{-1}\left\{s^{-\alpha} L\left\{\left(\frac{1}{2} x^{2}-\frac{1}{4}\right) u_{x x}\right\}\right\}$.

By applying the aforesaid homotopy perturbation method, we have:

$\sum_{n=0}^{+\infty} p^{n} u_{n}=2 x^{2}-1+p L^{-1}\left\{s^{-\alpha} L\left\{\left(\frac{1}{2} x^{2}-\frac{1}{4}\right) \sum_{n=0}^{+\infty} p^{n}\left(u_{n}\right)_{x x}\right\}\right\}$.

Equating the coefficient of the like power of $p$ on both sides in Eq. (104), we get:

$p^{0}: u_{0}(x, t)=2 x^{2}-1$,

$p^{1}: u_{1}(x, t)=L^{-1}\left\{s^{-\alpha} L\left\{\left(\frac{1}{2} x^{2}-\frac{1}{4}\right)\left(u_{0}\right)_{x x}\right\}\right\}$,

$p^{n}: u_{n}(x, t)=L^{-1}\left\{s^{-\alpha} L\left\{\left(\frac{1}{2} x^{2}-\frac{1}{4}\right)\left(u_{n-1}\right)_{x x}\right\}\right\}$,

where $n \in \square^{*}$.

Using the iteration formula (105), we obtain

$u_{0}(x, t)=2 x^{2}-1$,

$u_{1}(x, t)=\frac{2 x^{2}-1}{\Gamma(\alpha+1)} t^{\alpha}$,

$u_{2}(x, t)=\frac{2 x^{2}-1}{\Gamma(2 \alpha+1)} t^{2 \alpha}$,

$\vdots$

$u_{n}(x, t)=\frac{2 x^{2}-1}{\Gamma(n \alpha+1)} t^{n \alpha}$.

Finally, we approximate the analytical solution $u(x, t)$, by truncated series:

$u(x, t)=\sum_{n=0}^{+\infty} \frac{2 x^{2}-1}{\Gamma(n \alpha+1)} t^{n \alpha}$.

The terms of the decomposition series solution, for the special case $\alpha=1$, is given by:

$u(x, t)=\sum_{n=0}^{+\infty} \frac{2 x^{2}-1}{n !} t^{n}=\left(2 x^{2}-1\right) e^{t}$. 
which is an exact solution to the Eq. (35).

Figure 10.

We plotted these surfaces by using Maple software:

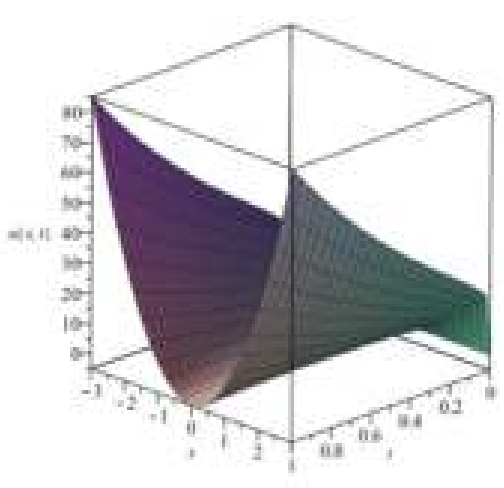

(a) $\mathrm{a}=0.5$

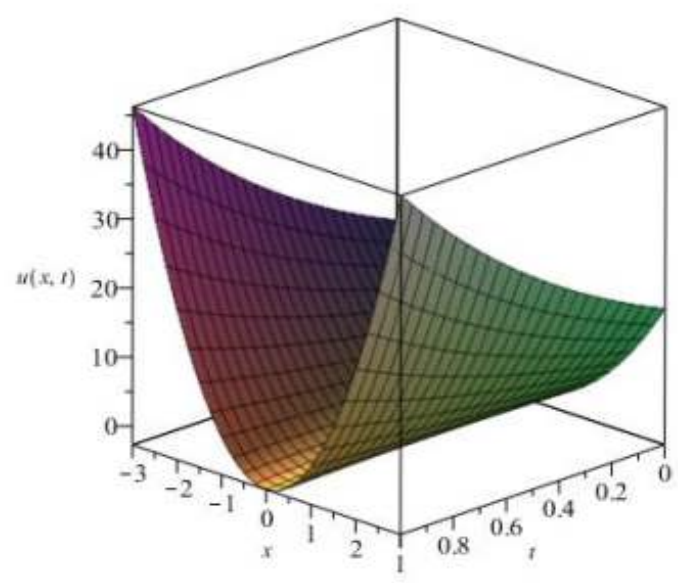

(c) Exact solution

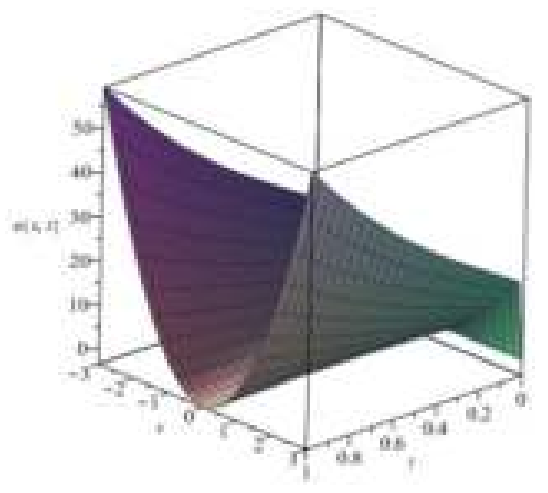

(b) $a=0.75$

Figure 10.1: Series approximation solution of Eq. (100), when $\alpha=0,5, \alpha=0,75$ with the first eleven terms and the Exact solution when $\alpha=1$.

Example 10.2. : Consider the following time-fractional equation with variable coefficients:

$$
\begin{cases}D_{* t}^{\alpha} u(x, y, t)+\frac{y^{2}-1}{2} u_{x x}-\frac{x^{2}+1}{2} u_{y y}=0, & t \succ 0 \\ 0 \prec \alpha \leq 1, \quad u(x, y, 0)=y^{2}-1, & t=0 .\end{cases}
$$

Taking the Laplace transform on both sides of Eq. (108), we get:

$$
L\left\{D_{* t}^{\alpha} u(x, y, t)\right\}=L\left\{\frac{x^{2}+1}{2} u_{y y}-\frac{y^{2}-1}{2} u_{x x}\right\} \text {. }
$$

An application of Eq. (11), yields:

$L\{u(x, y, t)\}=s^{-1}\left(y^{2}-1\right)+s^{-\alpha} L\left\{\frac{x^{2}+1}{2} u_{y y}-\frac{y^{2}-1}{2} u_{x x}\right\}$.

Applying the inverse Laplace transform of both sides in Eq. (110), we get:

$u(x, y, t)=y^{2}-1+L^{-1}\left\{s^{-\alpha} L\left\{\frac{x^{2}+1}{2} u_{y y}-\frac{y^{2}-1}{2} u_{x x}\right\}\right\}$. 
By applying the aforesaid homotopy perturbation method, we have:

$\sum_{n=0}^{+\infty} p^{n} u_{n}=y^{2}-1+p L^{-1}\left\{s^{-\alpha} L\left\{\frac{x^{2}+1}{2} \sum_{n=0}^{+\infty} p^{n}\left(u_{n}\right)_{Y Y}-\frac{y^{2}-1}{2} \sum_{n=0}^{+\infty} p^{n}\left(u_{n}\right)_{x x}\right\}\right\}$.

Equating the coefficient of the like power of $p$ on both sides in Eq. (104), we get:

$p^{0}: u_{0}(x, y, t)=y^{2}-1$,

$p^{1}: u_{1}(x, y, t)=L^{-1}\left\{s^{-\alpha} L\left\{\frac{x^{2}+1}{2}\left(u_{0}\right)_{y y}-\frac{y^{2}-1}{2}\left(u_{0}\right)_{x x}\right\}\right\}$,

$p^{n}: u_{n}(x, y, t)=L^{-1}\left\{s^{-\alpha} L\left\{\frac{x^{2}+1}{2}\left(u_{n-1}\right)_{y y}-\frac{y^{2}-1}{2}\left(u_{n-1}\right)_{x x}\right\}\right\}$,

where $n \in \square^{*}$.

Using the iteration formula (113), we obtain

$u_{0}(x, y, t)=y^{2}-1$,

$u_{1}(x, y, t)=\frac{x^{2}+1}{\Gamma(\alpha+1)} t^{\alpha}$

$u_{2}(x, y, t)=-\frac{y^{2}-1}{\Gamma(2 \alpha+1)} t^{2 \alpha}$,

$u_{3}(x, y, t)=-\frac{x^{2}+1}{\Gamma(3 \alpha+1)} t^{3 \alpha}$,

$\vdots$

$u_{2 n}(x, y, t)=(-1)^{n} \frac{y^{2}-1}{\Gamma(2 n \alpha+1)} t^{2 n \alpha}$,

$u_{2 n+1}(x, y, t)=(-1)^{n} \frac{x^{2}+1}{\Gamma((2 n+1) \alpha+1)} t^{(2 n+1) \alpha}$.

Finally, we approximate the analytical solution $u(x, y, t)$, by truncated series:

$u(x, y, t)=\left(y^{2}-1\right) \sum_{n=0}^{+\infty}(-1)^{n} \frac{t^{2 n \alpha}}{\Gamma(2 n \alpha+1)}+\left(x^{2}+1\right) \sum_{n=0}^{+\infty}(-1)^{n} \frac{t^{(2 n+1) \alpha}}{\Gamma((2 n+1) \alpha+1)}$.

The terms of the decomposition series solution, for the special case $\alpha=1$, is given by:

$u(x, y, t)=\left(x^{2}+1\right) \sin t+\left(y^{2}-1\right) \cos t$,

which is an exact solution to the Eq. (42)

Example 10.3. : Consider the following time-fractional equation with variable coefficients:

$\begin{cases}D_{* t}^{\alpha} u(x, y, z, t)=\frac{x^{2}}{12} u_{x x}+\frac{y^{2}}{24} u_{y y}+\frac{z^{2}}{24} u_{z z}, \quad t \succ 0 \\ 0 \prec \alpha \leq 1, \quad u(x, y, z, 0)=2 x^{3} y^{3} z^{3}, & t=0 .\end{cases}$

Taking the Laplace transform on both sides of Eq. (116), we get:

$L\left\{D_{* t}^{\alpha} u(x, y, z, t)\right\}=L\left\{\frac{x^{2}}{12} u_{x x}+\frac{y^{2}}{24} u_{y y}+\frac{z^{2}}{24} u_{z z}\right\}$.

An application of Eq. (11), yields:

$L\{u(x, y, z, t)\}=s^{-1}\left(2 x^{3} y^{3} z^{3}\right)+s^{-\alpha} L\left\{\frac{x^{2}}{12} u_{x x}+\frac{y^{2}}{24} u_{y y}+\frac{z^{2}}{24} u_{z z}\right\}$. 
Applying the inverse Laplace transform of both sides in Eq. (118), we get:

$u(x, y, z, t)=2 x^{3} y^{3} z^{3}+L^{-1}\left\{s^{-\alpha} L\left\{\frac{x^{2}}{12} u_{x x}+\frac{y^{2}}{24} u_{y y}+\frac{z^{2}}{24} u_{z z}\right\}\right\}$.

By applying the aforesaid homotopy perturbation method, we have:

$\sum_{n=0}^{+\infty} p^{n} u_{n}=2 x^{3} y^{3} z^{3}+p L^{-1}\left\{s^{-\alpha} L\left\{\frac{x^{2}}{12} \sum_{n=0}^{+\infty} p^{n}\left(u_{n}\right)_{x x}+\frac{y^{2}}{24} \sum_{n=0}^{+\infty} p^{n}\left(u_{n}\right)_{y y}+\frac{z^{2}}{24} \sum_{n=0}^{+\infty} p^{n}\left(u_{n}\right)_{z z}\right\}\right\}$.

Equating the coefficient of the like power of $p$ on both sides in Eq. (104), we get:

$p^{0}: u_{0}(x, y, z, t)=2 x^{3} y^{3} z^{3}$,

$p^{1}: u_{1}(x, y, z, t)=L^{-1}\left\{s^{-\alpha} L\left\{\frac{x^{2}}{12}\left(u_{0}\right)_{x x}+\frac{y^{2}}{24}\left(u_{0}\right)_{y y}+\frac{z^{2}}{24}\left(u_{0}\right)_{z z}\right\}\right\}$,

$\vdots$

$p^{n}: u_{n}(x, y, z, t)=L^{-1}\left\{s^{-\alpha} L\left\{\frac{x^{2}}{12}\left(u_{n-1}\right)_{x x}+\frac{y^{2}}{24}\left(u_{n-1}\right)_{y y}+\frac{z^{2}}{24}\left(u_{n-1}\right)_{z z}\right\}\right\}$,

where $n \in \square^{*}$.

Using the iteration formula (121), we obtain

$u_{0}(x, y, z, t)=2 x^{3} y^{3} z^{3}$,

$u_{1}(x, y, z, t)=\frac{2 x^{3} y^{3} z^{3}}{\Gamma(\alpha+1)} t^{\alpha}$,

$u_{2}(x, y, z, t)=\frac{2 x^{3} y^{3} z^{3}}{\Gamma(2 \alpha+1)} t^{2 \alpha}$,

$:$

$u_{n}(x, y, z, t)=\frac{2 x^{3} y^{3} z^{3}}{\Gamma(n \alpha+1)} t^{n \alpha}$.

Finally, we approximate the analytical solution $u(x, y, z, t)$, by truncated series:

$u(x, y, z, t)=\sum_{n=0}^{+\infty} \frac{2 x^{3} y^{3} z^{3}}{\Gamma(n \alpha+1)} t^{n \alpha}$.

The terms of the decomposition series solution, for the special case $\alpha=1$, is given by:

$u(x, y, z, t)=\sum_{n=0}^{+\infty} \frac{2 x^{3} y^{3} z^{3}}{n !} t^{n}=2 x^{3} y^{3} z^{3} e^{t}$.

which is an exact solution to the Eq. (49)

Example 10.4.: Consider the following time-fractional equation with variable coefficients:

$$
\left\{\begin{array}{l}
D_{* t}^{\alpha} u(x, t)=\frac{x^{2}-1}{2} u_{x x}, \quad t \succ 0 \\
1 \prec \alpha \leq 2, \quad u(x, 0)=x^{2}-1, \quad u_{t}(x, 0)=0, \quad t=0 .
\end{array}\right.
$$

Taking the Laplace transform on both sides of Eq. (124), we get:

$L\left\{D_{* t}^{\alpha} u(x, t)\right\}=L\left\{\frac{x^{2}-1}{2} u_{x x}\right\}$.

An application of Eq. (11), yields:

$L\{u(x, t)\}=s^{-1}\left(x^{2}-1\right)+s^{-\alpha} L\left\{\frac{x^{2}-1}{2} u_{x x}\right\}$. 
Applying the inverse Laplace transform of both sides in Eq. (126), we get:

$u(x, t)=x^{2}-1+L^{-1}\left\{s^{-\alpha} L\left\{\frac{x^{2}-1}{2} u_{x x}\right\}\right\}$.

By applying the aforesaid homotopy perturbation method, we have:

$\sum_{n=0}^{+\infty} p^{n} u_{n}=x^{2}-1+p L^{-1}\left\{s^{-\alpha} L\left\{\frac{x^{2}-1}{2} \sum_{n=0}^{+\infty} p^{n}\left(u_{n}\right)_{x x}(x, t)\right\}\right\}$.

Equating the coefficient of the like power of $p$ on both sides in Eq. (128), we get:

$$
\begin{aligned}
& p^{0}: u_{0}(x, t)=x^{2}-1, \\
& p^{1}: u_{1}(x, t)=L^{-1}\left\{s^{-\alpha} L\left\{\frac{x^{2}-1}{2}\left(u_{0}\right)_{x x}\right\}\right\}, \\
& \vdots \\
& p^{n}: u_{n}(x, t)=L^{-1}\left\{s^{-\alpha} L\left\{\frac{x^{2}-1}{2}\left(u_{n-1}\right)_{x x}\right\}\right\},
\end{aligned}
$$

where $n \in \square^{*}$.

Using the iteration formula (129), we obtain

$$
\begin{aligned}
& u_{0}(x, t)=x^{2}-1, \\
& u_{1}(x, t)=\frac{x^{2}-1}{\Gamma(\alpha+1)} t^{\alpha}, \\
& u_{2}(x, t)=\frac{x^{2}-1}{\Gamma(2 \alpha+1)} t^{2 \alpha}, \\
& \vdots \\
& u_{n}(x, t)=\frac{x^{2}-1}{\Gamma(n \alpha+1)} t^{n \alpha} .
\end{aligned}
$$

Finally, we approximate the analytical solution $u(x, t)$, by truncated series:

$u(x, t)=\sum_{n=0}^{+\infty} \frac{x^{2}-1}{\Gamma(n \alpha+1)} t^{n \alpha}$.

The terms of the decomposition series solution, for the special case $\alpha=2$, is given by:

$u(x, t)=\sum_{n=0}^{+\infty} \frac{x^{2}-1}{(2 n) !} t^{2 n}=\left(x^{2}-1\right) \cosh t$,

which is an exact solution to the Eq. (56). 
Figure 10.2:

We plotted these surfaces by using Maple software:

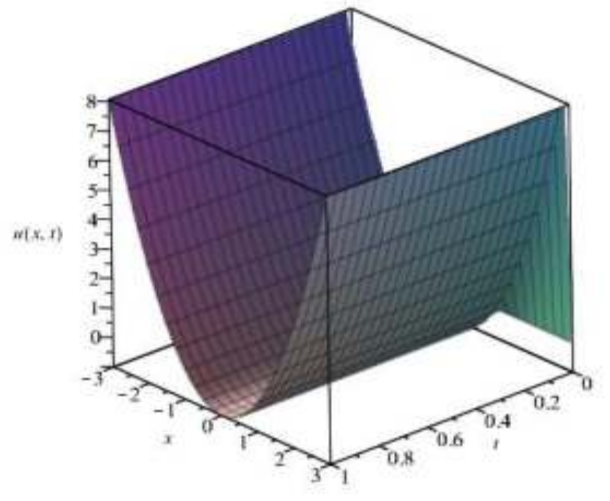

(a) $\alpha=1.5$

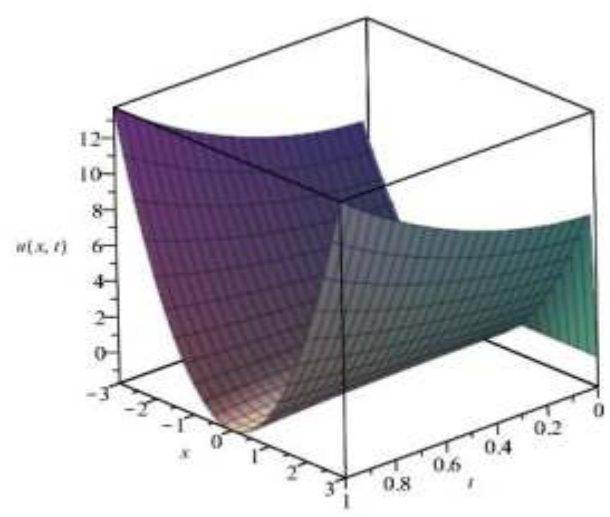

(b) $\alpha=1.75$

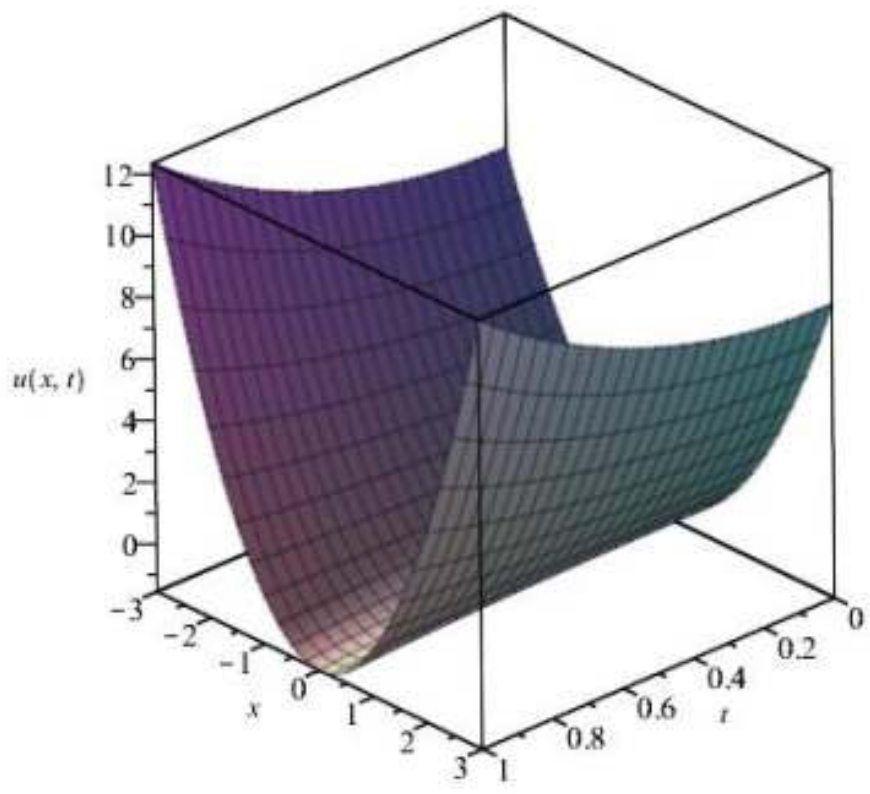

(c) Exact Solution

Figure 10.2: Series approximation solution of Eq. (124), when $\alpha=1,5, \alpha=1,75$ with the first eleven terms and the Exact solution when $\alpha=2$.

Example 10.5.:Consider the following time-fractional equation with variable coefficients:

$$
\begin{cases}D_{* t}^{\alpha} u(x, y, t)=\frac{1}{6} x^{2} u_{x x}+\frac{1}{6} y^{2} u_{y y}, \quad t \succ 0 \\ 1 \prec \alpha \leq 2, \quad u(x, y, 0)=0, & u_{t}(x, y, 0)=x^{3}+y^{3}, \quad t=0 .\end{cases}
$$

Taking the Laplace transform on both sides of Eq. (132), we get:

$$
L\left\{D_{* t}^{\alpha} u(x, y, t)\right\}=L\left\{\frac{1}{6} x^{2} u_{x x}+\frac{1}{6} y^{2} u_{y y}\right\} .
$$

An application of Eq. (11) yields:

$L\{u(x, y, t)\}=s^{-2}\left(x^{3}+y^{3}\right)+s^{-\alpha} L\left\{\frac{1}{6} x^{2} u_{x x}+\frac{1}{6} y^{2} u_{y y}\right\}$. 
Applying the inverse Laplace transform on both sides in Eq. (134), we get:

$u(x, y, t)=\left(x^{3}+y^{3}\right) t+L^{-1}\left\{s^{-\alpha} L\left\{\frac{1}{6} x^{2} u_{x x}+\frac{1}{6} y^{2} u_{y y}\right\}\right\}$.

By applying the aforesaid homotopy perturbation method, we have:

$\sum_{n=0}^{+\infty} p^{n} u_{n}=\left(x^{3}+y^{3}\right) t+p L^{-1}\left\{s^{-\alpha} L\left\{\frac{1}{6} x^{2} \sum_{n=0}^{+\infty} p^{n}\left(u_{n}\right)_{x x}+\frac{1}{6} y^{2} \sum_{n=0}^{+\infty} p^{n}\left(u_{n}\right)_{y y}\right\}\right\}$.

Equating the coefficient of the like power of $p$ on both sides in Eq. (136), we get:

$p^{0}: u_{0}(x, y, t)=\left(x^{3}+y^{3}\right) t$,

$p^{1}: u_{1}(x, y, t)=L^{-1}\left\{s^{-\alpha} L\left\{\frac{1}{6} x^{2}\left(u_{0}\right)_{x x}+\frac{1}{6} y^{2}\left(u_{0}\right)_{y y}\right\}\right\}$,

:

$p^{n}: u_{n}(x, y, t)=L^{-1}\left\{s^{-\alpha} L\left\{\frac{1}{6} x^{2}\left(u_{n-1}\right)_{x x}+\frac{1}{6} y^{2}\left(u_{n-1}\right)_{y y}\right\}\right\}$,

where $n \in \square^{*}$.

Using the iteration formula (137), we obtain

$$
\begin{aligned}
& u_{0}(x, y, t)=\left(x^{3}+y^{3}\right) t, \\
& u_{1}(x, y, t)=\frac{x^{3}+y^{3}}{\Gamma(\alpha+2)} t^{\alpha+1}, \\
& u_{2}(x, y, t)=\frac{x^{3}+y^{3}}{\Gamma(2 \alpha+2)} t^{2 \alpha+1}, \\
& \vdots \\
& u_{n}(x, y, t)=\frac{x^{3}+y^{3}}{\Gamma(n \alpha+2)} t^{n \alpha+1} .
\end{aligned}
$$

Finally, we approximate the analytical solution $u(x, y, t)$, by truncated series:

$$
u(x, y, t)=\sum_{n=0}^{+\infty} \frac{x^{3}+y^{3}}{\Gamma(n \alpha+2)} t^{n \alpha+1} \text {. }
$$

The terms of the decomposition series solution, for the special case $\alpha=2$, is given by:

$u(x, y, t)=\sum_{n=0}^{+\infty} \frac{x^{3}+y^{3}}{(2 n+1) !} t^{2 n+1}=\left(x^{3}+y^{3}\right) \sinh t$,

which is an exact solution to the Eq. (63).

Example 10.6. : Consider the following time-fractional equation with variable coefficients:

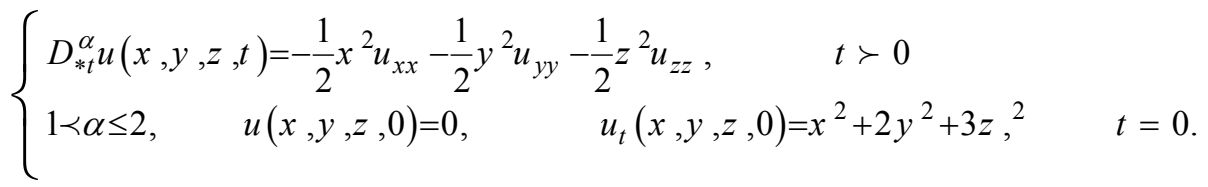

Taking the Laplace transform on both sides of Eq. (140), we get:

$$
L\left\{D_{* t}^{\alpha} u(x, y, z, t)\right\}=-L\left\{\frac{1}{2} x^{2} u_{x x}+\frac{1}{2} y^{2} u_{y y}+\frac{1}{2} z^{2} u_{z z}\right\} \text {. }
$$

An application of Eq. (11), yields:

$$
L\{u(x, y, z, t)\}=s^{-2}\left(x^{2}+2 y^{2}+3 z^{2}\right)-s^{-\alpha} L\left\{\frac{1}{2} x^{2} u_{x x}+\frac{1}{2} y^{2} u_{y y}+\frac{1}{2} z^{2} u_{z z}\right\} .
$$


Applying the inverse Laplace transform of both sides in Eq. (142), we get:

$$
u(x, y, z, t)=\left(x^{2}+2 y^{2}+3 z^{2}\right) t-L^{-1}\left\{s^{-\alpha} L\left\{\frac{1}{2} x^{2} u_{x x}+\frac{1}{2} y^{2} u_{y y}+\frac{1}{2} z^{2} u_{z z}\right\}\right\} \text {. }
$$

By applying the aforesaid homotopy perturbation method, we have:

$$
\sum_{n=0}^{+\infty} p^{n} u_{n}=\left(x^{2}+2 y^{2}+3 z^{2}\right) t-p L^{-1}\left\{s^{-\alpha} L\left\{\frac{1}{2} x^{2} \sum_{n=0}^{+\infty} p^{n}\left(u_{n}\right)_{x x}+\frac{1}{2} y^{2} \sum_{n=0}^{+\infty} p^{n}\left(u_{n}\right)_{y y}+\frac{1}{2} z^{2} \sum_{n=0}^{+\infty} p^{n}\left(u_{n}\right)_{z z}\right\}\right\} .(14
$$

Equating the coefficient of the like power of $p$ on both sides in Eq. (144), we get:

$$
\begin{aligned}
& p^{0}: u_{0}(x, y, z, t)=\left(x^{2}+2 y^{2}+3 z^{2}\right) t, \\
& p^{1}: u_{1}(x, y, z, t)=-L^{-1}\left\{s^{-\alpha} L\left\{\frac{1}{2} x^{2}\left(u_{0}\right)_{x x}+\frac{1}{2} y^{2}\left(u_{0}\right)_{y y}+\frac{1}{2} z^{2}\left(u_{0}\right)_{z z}\right\}\right\}, \\
& \vdots \\
& p^{n}: u_{n}(x, y, z, t)=-L^{-1}\left\{s^{-\alpha} L\left\{\frac{1}{2} x^{2}\left(u_{n-1}\right)_{x x}+\frac{1}{2} y^{2}\left(u_{n-1}\right)_{y y}+\frac{1}{2} z^{2}\left(u_{n-1}\right)_{z z}\right\}\right\},
\end{aligned}
$$

where $n \in \square^{*}$.

Using the iteration formula (145), we obtain

$$
\begin{aligned}
& u_{0}(x, y, z, t)=\left(x^{2}+2 y^{2}+3 z^{2}\right) t, \\
& u_{1}(x, y, z, t)=-\frac{x^{2}+2 y^{2}+3 z^{2}}{\Gamma(\alpha+2)} t^{\alpha+1}, \\
& u_{2}(x, y, z, t)=\frac{x^{2}+2 y^{2}+3 z^{2}}{\Gamma(2 \alpha+2)} t^{2 \alpha+1}, \\
& \vdots \\
& u_{n}(x, y, z, t)=(-1)^{n} \frac{x^{2}+2 y^{2}+3 z^{2}}{\Gamma(n \alpha+2)} t^{n \alpha+1} .
\end{aligned}
$$

Finally, we approximate the analytical solution $u(x, y, z, t)$, by truncated series:

$$
u(x, y, z, t)=\sum_{n=0}^{+\infty}(-1)^{n} \frac{x^{2}+2 y^{2}+3 z^{2}}{\Gamma(n \alpha+2)} t^{n \alpha+1} .
$$

The terms of the decomposition series solution, for the special case $\alpha=2$, is given by

$$
u(x, y, z, t)=\sum_{n=0}^{+\infty}(-1)^{n}\left(x^{2}+2 y^{2}+3 z^{2}\right) \frac{t^{2 n+1}}{(2 n+1) !}=\left(x^{2}+2 y^{2}+3 z^{2}\right) \sin t,
$$

which is an exact solution to the Eq. (70).

\section{CONCLUSION:}

In this paper, we have seen that the coupling of homotopy perturbation method (HPM) and the Laplace transform, proved very effective to solve certain type of partial and fractional partial differential equations.

The proposed algorithm (HPTM) is suitable for such problems and is very user friendly. The advantage of this method is its ability to obtain exact solutions of partial and fractional partial differential equations. The result obtained in the examples presented shows that this modified method is very powerful and efficient technique in finding exact solutions for wide classes of problems.

\section{ACKNOWLEDGEMENTS}

The authors would like to express their gratitude to the referee. We are very grateful for his help, his careful observations and his careful reading, which led to the improvement of the article.

\section{REFERENCES}

K. Abbaoui and Y. Cherruault, New ideas for proving convergence of decomposition methods Comput. Math. Appl. 29 (1995).pp. 103-108.

A. J. Al-Saif and D. A. Abood, TheHomotopy Perturbation Method for Solving K $(2,2)$ Equation, J. 
Of Basrah Researches (Sciences), Vol. 37.No. 4. C (2011).

A. S. J. AL-Saif and T. A. K. Hattim, Variational Iteration Method for Solving Some Models of Nonlinear Partial Differential Equations, Int. J. Pure

Appl. Sci. Technol., 4(1)(2011). pp. 30-40.

A. Ghorbani and J. Saberi-Nadja, perturbation Method for calculating adomian polynomials, International Journal of Nonlinear Sciences and Numerical Simulation, 8 (2007). pp. 229-232.

A. Ghorbani ,adomian polynomials: He polynomials, Choas Solitons Fractals, 39 (2009). pp. 14861492.

Ji-Huan He, Comparison of homotopy perturbation method and homotopy analysis method, Applied Mathematics and Computation 156 (2004). pp. 527-539.

J. H. He, Homotopy Perturbation Technique, comput. Methods Appl. Mech. Engrg, 178(1999). 257262.

J. H. He, A new perturbation technique which is also valid for large parameters, J. Sound Vib, 229 (2000). 1257-1263.

J. H. He, Application of homotopy perturbation method to nonlinear wave equations, Chaos Solitons

Fractals, 26(3) (2005). pp. 695-700.

J. H. He, A coupling method of Homotopy technique and perturbation technique for nonlinear problems, Int. J. Non-Linear Mech, 35 (2000). pp. 37-43.
J. $\mathrm{H}$. He and $\mathrm{He} \mathrm{JH}$, Homotopy perturbation method. Anew nonlinear technique. Appl. Math. Comput.135 (2003).pp. 73-79.

L. Jin, Homotopy Perturbation Method for Solving Partial Differential Equations with Variable Coefficients, Contemp. Math. Sciences, Volume 3 , no. 28 (2008).pp. 1395-1407.

Y. Khan and Q. Wu, Homotopy perturbation transform method for nonlinear equation using He's polynomials Computers and Mathematics with Applications. 61 (2011).pp. 1963-1967.

S. Kumar and A. Yildirim and Y. Khanc and L. Weid, Afractional model of the diffusion equation and its analytical solution using Laplace transform Sci. Iranica B, 19(4) (2012).pp. 1117-1123.

Joel L. Schiff, The laplaceTranform, Theory and Applications, Springer-Verlag, New York 1999

Murray R. Spiegel, laplaceTranforms, McGRAWHILL, New York 1965.

A. M. Wazwaz, A reliabe modification of Adomian decomposition method, Applied Mathematics and Computation, Volume 102 (1999).pp.77-86.

A. M. Wazwaz, Exact solutions for heat-like and wave-like equations with variable coefficients, AppliedMathematics and Computation, Volume 149 (2004).pp. 15-29.

D. Ziane and K. Belghaba and M. HamdiCherif, Fractional homotopy perturbation transform method for solving the time-fractional $\mathrm{KdV}$, $\mathrm{K}(2,2)$ and Burgers equations , Open Problems Compt. Math, Volume8,no.2 (2015). pp. 63-75. 